BULLETIN Bulletin hispanique

HISPANIQUE Université Michel de Montaigne Bordeaux

$112-2 \mid 2010$

Varia

\title{
Ouvrages reçus par le Bulletin Hispanique
}

\section{Dominique Breton}

\section{OpenEdition}

\section{Journals}

Édition électronique

URL : http://journals.openedition.org/bulletinhispanique/1304

DOI : 10.4000/bulletinhispanique.1304

ISSN : 1775-3821

\section{Éditeur}

Presses universitaires de Bordeaux

\section{Édition imprimée}

Date de publication : 31 décembre 2010

Pagination : 865-871

ISBN : 978-2-86781-709-0

ISSN : 0007-4640

Référence électronique

Dominique Breton, « Ouvrages reçus par le Bulletin Hispanique », Bulletin hispanique [En ligne], 112-2 |

2010, mis en ligne le 19 janvier 2013, consulté le 22 septembre 2020. URL : http://

journals.openedition.org/bulletinhispanique/1304; DOI : https://doi.org/10.4000/bulletinhispanique. 1304

Tous droits réservés 


\title{
OUVRAGES REÇUS PAR LE BULLETIN HISPANIQUE
}

\author{
I. - LitTÉRATURE, ÉDitions ET ÉTUDES
}

\section{Espagne}

Alarcón Sierra (Rafael), Una Rana viajera: Las crónicas y los libros de viaje de Julio Camba. - España, Renacimiento, 2010, 175 p. (Colección Iluminaciones, Filología, crítica y ensayo, 59) : bibliografía - ISBN 97884-8472-563-3.

Ángeles Naval (María), Cuestión de memoria. Estudios sobre Ramón J. Sender, Luis Cernuda y Francisco Ayala. - Zaragoza, Institución Fernando el Católico (CSIC), 2010, 158 p. (Colección de letras). - ISBN 84-9911043-1.

Benito Pérez Galdós en el cine mexicano: literatura y cine. John H. Sinnigen (ed.). - México, Universidad Nacional Autónoma, 2008, 364 p. : ilustraciones, bibliográficas (colección Miradas en la oscuridad) - ISBN 978-607-2-00089-6.

Cavillac (Michel), Guzmán de Alfarache y la novela moderna. Francisco Rico (prólogo). - Madrid, Casa de Velázquez, 2010, 304 p. : bibliografía, índice onomástico, índice de materias, índice general (Bibliothèque de la Casa de Velázquez, volume 44) - ISBN 978-84-96820-39-5 - ISSN 0213-9758.

Cruickshank (DonW.), Don Pedro Calderón. - Cambridge, University Press, 2009, 471 p. : appendix: Calderon's dramatic works mentioned in this book, notes, bibliography, index of Calderon's works, general index, illustrations - ISBN 978-0-521-76515-2.

Egido (Aurora), El águila y la tela: Estudios sobre San Juan de la Cruz y Santa Teresa de Jesuis. - España, José J. de Olañeta, UIB, 2010, 295 p. (Medio Maravedí,15) - ISBN 978-84-9716-644-7.

El Quijote en América. Friedhelm Schmidt-Welle e Ingrid Simson. Amsterdam - New-York, Rodopi, 2010, 330 p. (Colección hispánica de

Bulletin Hispanique, Tome 112, nº 2 - décembre 2010 - p. 865 à 871. 
Flandes y Países Bajos, Foro Hispánico, 40) - ISBN 978-90-420-30510 - ISSN 0925-8620.

El teatro según Lope de Vega. 2 vols. Felipe B. Pedraza Jiménez, Milagros Rodríguez CÁceres (Estudio, selección, edición de los textos e índices). Mar Zubieta (Edición y documentación gráfica) - Madrid, Compañía Nacional de Teatro Clásico, 2009, 186 p. : fotografías e ilustraciones, índice de nombres propios y materias, índice general, ficha artística de los montajes (Cuadernos de Teatro Clásico, 25). - ISSN 0214-1388 NIPO 556-09-049-X.

La Historia de Francia en la Literatura Española. Amenaza o modelo. Mercè Boixareu y Robin Lefere (Coordinadores). - Madrid, Castalia, 2009, 796 p. : ilustraciones, apéndice documental, índice onomástico y de textos del corpus - ISBN 978-84-9740-275-0.

Lesage (Alain-René), Euvres complètes. Christelle Bahier-Porte et Pierre Brunel (dir.). Tome 8. Euvres romanesque IV. Histoire d'Estévanille Gonzalez surnommé le garçon de bonne humeur. Cécile Cavillac (édition critique). Le Bachelier de Salamanque ou les mémoires de Don Chérubin de la Ronda. Guiomar Hautceur (édition critique) - Paris, Honoré Champion, 2010, 824 p. : annexes, bibliographie, index des noms de personnages, index des auteurs et des œuvres cités (Sources classiques Collection dirigée par Philippe Sellier, 93) - ISBN 978-2-7453-1870-1 - ISSN 1169-2936.

Lesage (Alain-René), Euvres complètes. Christelle Bahier-Porte et Pierre Brunel (dir.). Tome 11. Euvres "adaptées ». III Histoire de Guzmán d'Alfarache. Cécile et Michel Cavillac (édition critique). 1 vol. - Paris, Honoré Champion, 2010, 592 p. : bibliographie, index des auteurs et des œuvres, index des noms de personnages, glossaire (Sources classiques - Collection dirigée par Philippe Sellier, 99) - ISBN 978-2-7453-1953-1 - ISSN 1169-2936.

Los conceptos de Gracián. Tercer Coloquio Internacional sobre Baltasar Gracián en ocasión de los 350 años de su muerte (Berlin, 27-29 de noviembre de 2008). Sebastian Neumeister (ed.). - Berlin, Tranvía. Verlag Walter Frey, 2010, 281 p. - ISBN 978-3-938944-40-0.

Olmo Iturriarte (Almudena de), Francisco Bejarano: una poética de la melancolía. - Illes Balears, Universitat, UIB, 2009, 151 p. : bibliografía (poetas y poéticas) - ISBN 978-84-8384-143-3.

Presencia de la tradición en la literatura española del Siglo de Oro. Natalia Fernández Rodríguez (Ed.). - Bellaterra, Universitat Autònoma de Barcelona, 2010, 382 p. : índice onomástico - ISBN 978-84-613-9649-8. Valdivia Martín (Pablo), La vereda indecisa. El viaje hacia la literatura de 
Federico García Lorca. - Granada, Diputación, 2009, 350 p. : bibliografía, ilustraciones (Colección Genil de literatura, 56). - ISBN 978-84-7807479-2.

Vilaseca (David), Queer Events: Post-deconstructive Subjectivities in Spanish Writing and Film 1960s-1990s. - Liverpool, University Press, 2010, 272 p. : bibliography, index (Contemporary Hispanic and Lusophone Studies, 4) - ISBN 978-1-84631-467-4.

VIVAR (Francisco), Don Quijote frente a los caballeros de los tiempos modernos. Salamanca, Universidad, 2009, 178 p. : bibliografía (Estudios filológicos, 325) - ISBN 978-84-7800-255-9.

\section{Amérique Latine}

Benito Pérez Galdós en el cine mexicano: literatura y cine. John H. Sinnigen (ed.). - México, Universidad Nacional Autónoma, 2008, 364 p. : ilustraciones, bibliográficas (colección Miradas en la oscuridad) - ISBN 978-607-2-00089-6.

Bonilla Cerezo (Rafael), Dos gauchos retrucadores. Nueva lectura del Fausto de Estanislao del Campo. Prólogo de Teodosio Fernández. - Alicante, Universidad, 2010, 222 p. : bibliografía, ilustraciones de Belén Abad de los Santos, cuadernos publicados (Cuadernos de América sin nombre, 26). - ISBN 978-84-9717-121-2.

Fridman (Viviana), Ecriture et identité juive en Argentine dans la transition démocratique. - Paris, Honoré Champion, 2010, 296 p. : bibliographie, index (Bibliothèque de Littérature générale et comparée, 83). - ISBN 978-2-7453-1889-3 - ISSN 1262-2850.

Mora (Carmen de), Escritura e Identidad Criollas: El Carnero, Cautiverio feliz e Infortunios de Alonso Ramírez. - Amsterdam - New York, Rodopi, 2010, 348 p. : bibliografía, índice de nombres (Colección hispánica de Flandes y Países Bajos, Foro Hispánico, 38). - ISBN 978-90-420-30138 - E-Book ISBN : 978-90-420-3014-5 - ISSN 0925-8620.

\section{Europe}

Pajares Infantes (Eterio), La traducción de la novela inglesa del siglo XVIII. Fernando Galván (ed.). - Vitoria, Portal , 2010, 502 p. : bibliografía, anexos - ISBN 978-84-937075-4-5.

Parallelismen, Parallélismes, Paralelismos. Mélanges de littérature et d'analyse culturelle offerts à Peter Fröhlicher. Marie Burkhardt, Annatina Platter, and Alain Schorderet (eds.). - Tübingen, Gunter Narr Verlag, 2009, 320 p. - ISBN 978-3-8233-6528-0. 
Shakespeare and His Authors. Critical Perspectives on the Authorship Question. William Leahy (ed.). - London/New York, Continuum, 2010, p.180 : bibliography, index - ISBN 978-0-8264-2611-6 (paperback) - 978-08264-3684-9 (hardback).

Shakespeare and Moral Agency. Michael D. Bristol (ed.). - London/New York, Continuum, 2010, 224 p. : Works Cited, Index - ISBN 978-08264-4676-3.

\section{II. - LinguistiQUe, TRADUCTION, TRADUCTOLOGIE}

Diccionario histórico de la traducción en España. Francisco LAFARGa y Luis Pegenaute (ed.). - Madrid, Gredos, 2009, 1192 p. - ISBN 978-84249-3626-6.

Estudios de lingüistica: Investigaciones lingüisticas en el siglo XXI. Juan Luis Jiménez Ruiz, Larissa TimofeEva (eds.). - Alicante, Universidad (ELUA), 2009, 271 p. - ISBN 978-84-930403-9-0 - ISSN 0212-7636.

La concordance des temps. Moyen Âge et Époque moderne. Gilles LuQueT (éd.) avec la collaboration de Virginie Dumanoir et Gabriel Le Tallec. Paris, Presses Sorbonne Nouvelle, 2010, p. 220. : présentation des auteurs, index des auteurs cités, index des notions. - ISBN 978-2-87854-468-8.

Lesage (Alain-René), Euvres complètes. Christelle Bahier-Porte et Pierre Brunel (dir.). Tome 8. Euvres romanesque IV. Histoire d'Estévanille Gonzalez surnommé le garçon de bonne humeur. Cécile Cavillac (édition critique). Le Bachelier de Salamanque ou les mémoires de Don Chérubin de la Ronda. Guiomar Hautceeur (édition critique) - Paris, Honoré Champion, 2010, 824 p. : annexes, bibliographie, index des noms de personnages, index des auteurs et des œuvres cités (Sources classiques Collection dirigée par Philippe Sellier, 93) - ISBN 978-2-7453-1870-1 - ISSN 1169-2936.

Lesage (Alain-René), Euvres complètes. Christelle Bahier-Porte et Pierre Brunel (dir.). Tome 11. CEuvres " adaptées ». III Histoire de Guzmán d'Alfarache. Cécile et Michel Cavillac (édition critique). 1 vol. - Paris, Honoré Champion, 2010, 592 p. : bibliographie, index des auteurs et des œuvres, index des noms de personnages, glossaire (Sources classiques - Collection dirigée par Philippe Sellier, 99) - ISBN 978-2-7453-1953-1 - ISSN 1169-2936. 
Morphologie et syntaxe de l'espagnol. Méthodes d'approche. Gilles Luquet (éd.). - Paris, Presses Sorbonne Nouvelle, 2010, 276 p. : présentation des auteurs, index des auteurs cités, index des notions. - ISBN 978-287854-469-5.

$$
\text { III. - Histoire - CULTURE - SOCIÉTÉ }
$$

\section{Espagne}

Diccionario histórico de la traducción en España. Francisco Lafarga y Luis Pegenaute (ed.). - Madrid, Gredos, 2009, 1192 p. - ISBN 978-84249-3626-6.

L'Espagne en 1808. Régénération ou révolution? Gérard Dufour et Elisabel LARRIBa (dir.). - Aix-en-Provence, Université, 2009, 324 p. (collection Le temps de l'Histoire) : index onomastique - ISBN 978-2-85399743-0.

Marín (Manuela), Puente (Cristina de la), Rodríguez Mediano (Fernando), Pérez Alcalde (Juan Ignacio), Los epistolarios de Julián Ribera Tarragó y Miguel Asín Palacios. Introducción, catálogo e indices. - Madrid, CSIC, 2009, 991 p. : bibliografía, índices de nombres de personas, de topónimos y de publicaciones periódicas mencionados en EJR y EMA (Estudios árabes e islámicos: monografías 16) - ISBN 97884-00-08921.4.

RojInsky (David), Companion to Empire. A Genealogy of the Written Word in Spain and New Spain, c. 550-1550. - Amsterdam - New York, Rodopi, 2010, 300 p. : bibliography, index (Colección hispánica de Flandes y Países Bajos, Foro Hispánico, 37) - ISBN 978-90-420-2866-1 - E-Book ISBN 978-90-420-2867-8 - ISSN 0925-8620.

Smith (Paul Julian), Spanish screen fiction. Between cinema and television. Liverpool, University Press, 2009, 200 p. (Contemporary Hispanic and Lusophone Cultures) : index, illustrations - ISBN 978-1-84631-201-4 cased - ISBN 978-1-84631-202-1 limp.

Vilaseca (David), Queer Events: Post-deconstructive Subjectivities in Spanish Writing and Film 1960s-1990s. - Liverpool, University Press, 2010, 272 p. : bibliography, index (Contemporary Hispanic and Lusophone Studies, 4) - ISBN 978-1-84631-467-4. 


\section{Amérique Latine}

Benito Pérez Galdós en el cine mexicano: literatura y cine. John H. Sinnigen (ed.). - México, Universidad Nacional Autónoma, 2008, 364 p. : ilustraciones, bibliográficas (colección Miradas en la oscuridad) - ISBN 978-607-2-00089-6.

Diccionario histórico de la traducción en España. Francisco LAFARGa y Luis Pegenaute (ed.). - Madrid, Gredos, 2009, 1192 p. - ISBN 978-84249-3626-6.

El Quijote en América. Friedhelm Schmidt-Welle e Ingrid Simson. Amsterdam - New-York, Rodopi, 2010, 330 p. (Colección hispánica de Flandes y Países Bajos, Foro Hispánico 40) - ISBN 978-90-420-3051-0 - ISSN 0925-8620.

Fridman (Viviana), Ecriture et identité juive en Argentine dans la transition démocratique. - Paris, Honoré Champion, 2010, 296 p. : bibliographie, index (Bibliothèque de Littérature générale et comparée, 83). - ISBN 978-2-7453-1889-3 - ISSN 1262-2850.

Ilustración en el Mundo Hispánico: preámbulo de las Independencias. Milena Koprivitza Acuña, Manuel Ramos Medina, María Cristina Torales Pacheco, José María Urkía Echave, Sabino Yano Bretón (eds.). - Tlaxcala, Gobierno de Estado Tlaxcala, Instituto Tlaxcalteca de la Cultura - México, Universidad Iberoamericana, 2009, 534 p. - ISBN 978-607-95355-0-6.

Lawrance (Jeremy), Spanish Conquest Protestant Prejudice: Las Casas and the Black Legend. - United Kingdom, Critical, Cultural and communications Press, 2009, 64 p. : figures, works cited (Monographs in Post-Conflict Cultures) - ISBN (UK) 978-1-905510-23-8 - ISBN (USA) 978-160271-020-7.

Mémoire et découvertes : quels paradigmes? Actes du colloque international organisé à Nancy (15,16 et 17 novembre 2007. Mary-Nelly FoulignY et Marie Roig Miranda (Dir.). Nancy, Université de Nancy II, 2009, 417 p. (collection Europe XVI-XVII, 13). - ISBN 978-2-917030-028 - ISSN 1623-880X.

Mora (Carmen de), Escritura e Identidad Criollas: El Carnero, Cautiverio feliz e Infortunios de Alonso Ramírez. - Amsterdam - New York, Rodopi, 2010, 348 p. : bibliografía, índice de nombres (Colección hispánica de Flandes y Países Bajos, Foro Hispánicos, 38). - ISBN 978-90-420-30138 - E-Book ISBN : 978-90-420-3014-5 - ISSN 0925-8620. 
Rojinsky (David), Companion to Empire. A Genealogy of the Written Word in Spain and New Spain, c. 550-1550. - Amsterdam - New York, Rodopi, 2010, 300 p. : bibliography, index (Colección hispánica de Flandes y Países Bajos, Foro Hispánico, 37) - ISBN 978-90-420-2866-1 E-Book ISBN 978-90-420-2867-8 - ISSN 0925-8620.

Saberes y sabores en México y el Caribe. Rita De Maeseneer y Patrick Collard (eds.), Kim Huyge (col.). - Amsterdam - New York, Rodopi, 2010, 356 p. : ilustraciones, notas bibliográficas sobre los autores (Colección hispánica de Flandes y Países Bajos, Foro Hispánicos, 39). - ISBN 97890-420-3044-2 - ISSN 0925-8620.

Ward (Thomas), Buscando la Nación Peruana. Matto de Turner, Cabello de Carbonera, González Prada, Riva-Agüero, Ricardo Palma, José Carlos Mariátegui, José María Arguedas Lima: Editorial Horizonte, 2009, 340 p. : Bibliografía, Abreviaturas usadas, (Pensamiento Peruano, 7) - ISBN 978-9972-699-53-5.

\section{Europe}

La Historia de Francia en la Literatura Española. Amenaza o modelo. Mercè Boixareu y Robin Lefere (Coordinadores). - Madrid, Castalia, 2009, 796 p. : ilustraciones, apéndice documental, índice onomástico y de textos del corpus - ISBN 978-84-9740-275-0.

Mémoire et découvertes : quels paradigmes? Actes du colloque international organisé à Nancy (15,16 et 17 novembre 2007. Mary-Nelly FoulignY et Marie Roig Miranda (Dir.). Nancy, Université de Nancy II, 2009, 417 p. (collection Europe XVI-XVII, 13). - ISBN 978-2-917030-028 - ISSN 1623-880X.

Dominique Breton 